\title{
Solid-State NMR Spectroscopy as a Tool Supporting Optimization of MALDI-TOF MS Analysis of Polylactides
}

\author{
Anna Sroka-Bartnicka, Sebastian Olejniczak, Marek Sochacki, \\ Tadeusz Biela, and Marek J. Potrzebowski \\ Centre of Molecular and Macromolecular Studies, Polish Academy of Sciences, Lodz, Poland
}

We report systematic structural studies of poly(L-lactide) (PLLA) employing matrix-assisted laser desorption/ionization time-of-flight (MALDI-TOF) and solid-state NMR spectroscopy. ${ }^{13} \mathrm{C}$ cross polarization magic angle spinning (CP/MAS) NMR data for 1,8-dihydroxy-9anthracenone (DT), 2,5-dihydroxybenzoic acid (DHB), 2-(4-hydroxyphenylazo)-benzoic acid (HABA), and trans-3-indoleacrylic acid (IAA), four matrices commonly used in MALDI-TOF analysis of polymers, were analyzed to test the influence of crystallization conditions (solvent, inorganic salt) on sample morphology. ${ }^{13} \mathrm{C}$ principal elements of chemical shift tensor (CST) and line-shape analyses were employed to study of the nature of hydrogen bonding and to evaluate the crystallinity and amorphicity of the pure polymer. NMR parameters for PLLA were compared with data for polylactide crystallized with the four matrices under different conditions with the addition of two inorganic salts as cationization agents. This study revealed that the semicrystalline structure of the polymer does not change when it is embedded in the matrix. (J Am Soc Mass Spectrom 2009, 20, 67-72) ( 2009 Published by Elsevier Inc. on behalf of American Society for Mass Spectrometry

$I$ n recent years matrix-assisted laser desorption/ionization time-of-flight mass spectrometry (MALDI-TOF MS) has become an important mass spectrometric technique for the analysis of polymers [1]. The usefulness of this method for determination of molecular mass, molecular mass distributions [2], and end groups of synthetic polymers has been reported in a number of excellent reviews [3]. As discussed in many papers, the proper choice of matrix and the method of sample preparation [4-7] are two factors that crucially influence the quality of mass spectra, and these issues remain the most difficult part of the MALDI analysis. Numerous research groups have examined this problem and have found correlations between crystal and/or molecular structure of the matrices and the MALDI spectral data [8]. Schubert's group has contributed important findings related to the choice of solvent for sample crystallization, matrix functional groups arrangement, the influence of degree of crystallinity on spectroscopic response, etc. [9]. On the other hand, Hoteling et al. found that the best MALDI spectra with respect to $\mathrm{S} / \mathrm{N}$ ratio were obtained when matrix and polymer had similar retention times in a gradient HPLC experiment [10]. Thus, no clear criteria have emerged that enable the a priori prediction of the optimal conditions for matrix-polymer sample preparation even

Address reprint requests to Dr. M. J. Potrzebowski, Centre of Molecular and Macromolecular Studies, Polish Academy of Sciences, Sienkiewicza 112, 90-363 Lodz, Poland. E-mail: marekpot@cbmm.lodz.pl though various experimental techniques in different phases have been employed to account for the complexity of these processes.

In the present study, our goal was to improve our understanding of all aspects that are important for proper preparation of samples for MALDI-TOF measurement. Since the most important step of analysis is solid-to-gas-phase transition, we focused our attention on the solid-state effects, and employed solid-state NMR spectroscopy as the technique of choice. Herein, we report systematic NMR studies of matrices commonly used in MALDI-TOF MS, including 1,8-dihydroxy-9anthracenone (DT), 2,5-dihydroxybenzoic acid (DHB), 2-(4hydroxyphenylazo)-benzoic acid (HABA), and trans-3indoleacrylic acid (IAA). We also investigated the influence of the cationization agents $\mathrm{NaI}, \mathrm{NaCl}$, and $\mathrm{LiCl}$ on molecular packing and crystallinity of the samples. Finally, we examined analytical systems prepared with these matrix materials, cationization agents, and the polymer by means of solid-state NMR (SS NMR).

In this project, as the model polymer (Scheme 1) we have used poly(L-lactide) (PLLA) with dipentaerythritol (DPE) core group and molecular mass of $3 \mathrm{kDa}(\mathbf{1})$. The choice of the DPE group as an end group of PLLA permitted the possibility for synthesis of star-shape polymers with unique functional properties. The current work is part of a larger project related to the synthesis and characterization of new biodegradable materials, which have attracted increasing interest over
(C) 2009 Published by Elsevier Inc. on behalf of American Society for Mass Spectrometry. 1044-0305/09/\$32.00

doi:10.1016/j.jasms.2008.09.013
Published online September 23, 2008

Received May 21, 2008 Revised September 5, 2008 Accepted September 5, 2008 
<smiles>CC(C(=O)OC(C)(C)C)C(C)(C)O</smiles>

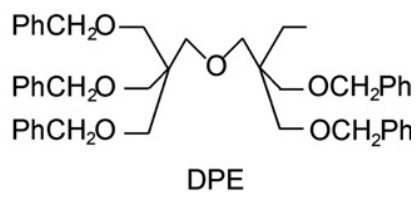

Scheme 1. Poly(L-lactide) (PLLA) with dipentaerythritol (DPE) core.

the past two decades in fundamental research and also in the chemical industry.

\section{Experimental}

The preparation of poly(L-lactide) (PLLA) with the dipentaerythritol (DPE) core group (1) has been described elsewhere [11]. Matrices 1,8-dihydroxy-9-anthracenone (DT), 2,5-dihydroxybenzoic acid (DHB), 2-(4-hydroxyphenylazo)-benzoic acid (HABA), trans-3indoleacrylic acid (IAA), and the cationization agents $\mathrm{NaI}, \mathrm{NaCl}, \mathrm{LiCl}$, and $\mathrm{KCl}$ were purchased from Sigma Aldrich and used without further purification. The polymer and the matrices were dissolved in tetrahydrofuran 99\% (THF; POCh, Gliwice, Poland) at a concentration of $10 \mathrm{mg} / \mathrm{mL}$. Solutions of the cationization agents were prepared at the concentration of $10 \mathrm{mg} / \mathrm{mL}$ in THF $(\mathrm{LiCl})$, acetone $(\mathrm{NaI})$, or water $(\mathrm{NaCl}, \mathrm{KCl})$. Samples were prepared by mixing the polymer solutions, matrix solutions, and the cationization agent solutions in the ratio 1:10:1 ( $\mathrm{vol} / \mathrm{vol} / \mathrm{vol})$. An aliquot (1 $\mu \mathrm{L}$ ) of this mixture was placed on the sample plate and allowed to dry. For each sample, three spots ( $1 \mu \mathrm{L}$ each) were applied to the sample plate beside each other. For each spot, three summed spectra were recorded. Each summation spectrum represented 10 repeated spectra from 25 different points on the sample spot ( 25 points $\times$ 10 spectra $=250$ spectra) and this procedure was repeated twice. The measurements were obtained by using the automatic mode of operation.

Mass spectra were recorded on a Voyager Elite mass spectrometer (PerSeptive Biosystems Inc., Framingham, $\mathrm{MA})$, equipped with nitrogen laser $(337 \mathrm{~nm})$. Acceleration voltage of $20 \mathrm{kV}$ and delayed extraction were used in all cases. Spectra were obtained in the linear, positive ion mode. All spectra, resolutions, and signal-to-noise $(\mathrm{S} / \mathrm{N})$ ratios were acquired, calculated and processed by Data Explorer ver. 4 program (Applied Biosystems, Foster City, CA).

For the screening, the instrument settings were: energy of laser beam, slighty above threshold level of 1800 a.u., at 1900 or 2000 a.u.; delay time, $100 \mathrm{~ns}$; acceleration voltage, $20,000 \mathrm{~V}$; acquisition mass range, 700 to 15,000 ; low mass gate, 500 ; grid voltage, $92.1 \%$; guide wire, $0.02 \%$.

Samples used in ${ }^{13} \mathrm{C}$ SS NMR studies were prepared as were the samples used in MALDI analysis, except that the amount of material used was increased to 200 $\mathrm{mg}$. The solid-state cross-polarization magic angle spinning (CP MAS) experiments were performed on a Bruker Avance DSX 300 (Bruker BioSpin GmbH, Rhein-
stetten/Karlsruhe) operating at $75.47 \mathrm{MHz}$ for ${ }^{13} \mathrm{C}$, equipped with a MAS probe head using $4 \mathrm{~mm}$ and 7 $\mathrm{mm} \mathrm{ZrO}_{2}$ rotors for ${ }^{13} \mathrm{C}$. Glycine was used for setting the Hartmann-Hahn condition and adamantane was used as a secondary chemical shift reference, $\delta=38.48 \mathrm{ppm}$ and $29.46 \mathrm{ppm}$ from external TMS [12]. The conventional spectra were recorded with a proton 90 degree pulse length of $3.5 \mu \mathrm{s}$ and a contact time of $1 \mathrm{~ms}$. The repetition delay was $10 \mathrm{~s}$ and the spectral width was $25 \mathrm{kHz}$. The FID signals were accumulated with a time domain size of $2 \mathrm{~K}$ data points. The RAMP shape pulse [13] was used during the cross-polarization and TPPM decoupling [14] with $\tau_{\mathrm{p}}=6.8 \mu \mathrm{s}$ and a phase angle of $20^{\circ}$ during the acquisition. The cross-polarization efficiency was measured with contact times between $10 \mu \mathrm{s}$ and $12 \mathrm{~ms}$. The spectral data were processed using the WIN-NMR program [15].

\section{Results and Discussion}

\section{MALDI TOF Measurements}

Polylactides belong to the class of polyesters for which DHB is most frequently used as a matrix in the MALDITOF MS measurements [1, 3]. Unfortunately, in the case of the polylactide under investigation, with the PLLA chain directly attached to the dipentaerythritol core (DPE), the mass spectra recorded using DHB were unsatisfactory due to poor resolution and low signal to noise $(\mathrm{S} / \mathrm{N})$ ratio. This unexpected result prompted us to examine the applicability of other matrices commonly used in the analysis of polymers, namely 1,8-dihydroxy-9-anthracenone (DT), 2-(4hydroxyphenylazo)-benzoic acid (HABA), and trans-3indoleacrylic acid (IAA).

We recorded MALDI-TOF spectra for $\mathbf{1}$ with DT and HABA matrices, using $\mathrm{NaI}$ as the cationization agent in both cases (Figure 1). The most distinctive differences
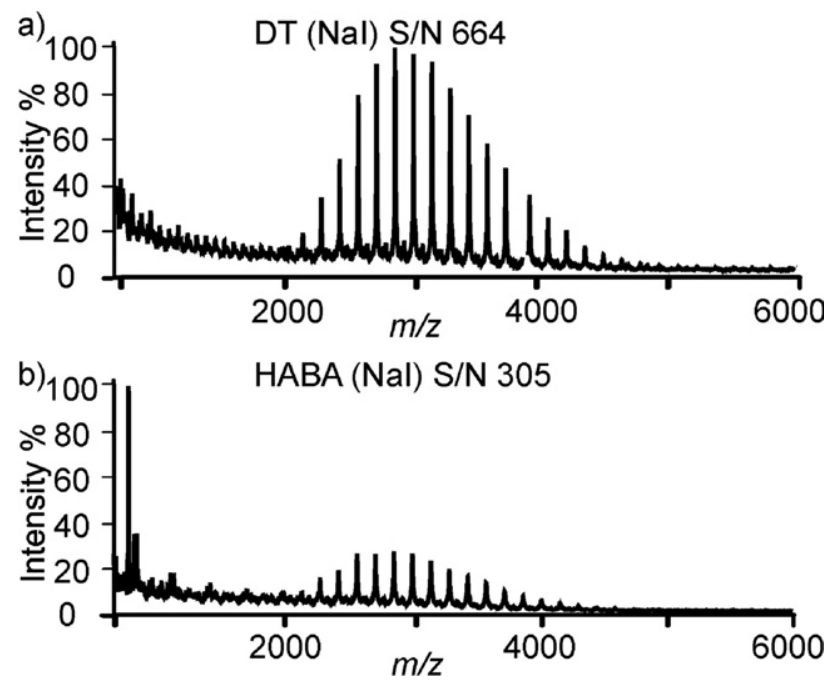

Figure 1. Comparison of best and worse quality MALDI-TOF mass spectra of 1, recorded in linear mode and obtained at laser beam energy of 2000 a.u. (a) Matrix, DT; cationization agent, NaI. (b) Matrix, HABA; cationization agent, $\mathrm{NaI}$. 
between the spectra were the intensities of the peaks, expressed by the $\mathrm{S} / \mathrm{N}$ ratio. While the distribution of signals in both cases is remarkably similar, the HABA matrix was evidently not the proper choice for $\mathbf{1}$ and gave unsatisfactory results.

We compared two parameters to assess the quality of the mass spectra: resolution and signal-to-noise $(\mathrm{S} / \mathrm{N})$ ratio. Both parameters were calculated for the highest peak in the spectrum, which corresponds to the cationized oligomer of highest concentration in the polymer.

The quality of MALDI-TOF spectra strongly depends on the compatibility of the cationization agent with polymer system [10]. Cationization agents are added to the polymer sample to facilitate formation of adducts (e.g., $\mathrm{M}+\mathrm{Na}$ ) in the gas phase. In this project, we investigated the influence of salts on MALDI-TOF results by testing $\mathrm{KCl}, \mathrm{NaCl}, \mathrm{LiCl}$, and $\mathrm{NaI}$. Samples for MALDI analysis were prepared in the volume ratio of polymer:matrix:salt equal to $1: 10: 1$, proportions that gave the highest intensity of peaks. Our preliminary study indicated that the best results were obtained with lithium chloride and sodium iodide, and for subsequent measurements, these two cationization agents were used.

The choice of solvent used for sample crystallization has significant influence on the quality of the spectra. As recently discussed by Meier et al. [9], using the same solvent for preparing solutions of polymer, matrix, and inorganic salt gives the best result, but binary solvent systems can be used as long as both are compatible. In our case, tetrahydrofuran (THF) fulfilled all requirements.

We next tested the influence of the energy of the laser beam on the quality of MALDI TOF spectra. As summarized in Table $1, \mathrm{~S} / \mathrm{N}$ strongly depends on the cationization agent. The best quality spectra were obtained for DT as the matrix in conjunction with $\mathrm{NaI}$ as the cationization reagent. Interestingly, this sample S/N does not depend on the energy of the laser beam within the measured range. The same matrix with $\mathrm{LiCl}$ gave much poorer results. On the basis of collected data, a ranking of the matrices (cationization agent), from best to worst, was determined as:

$$
\begin{aligned}
& \mathrm{DT}(\mathrm{NaI})>\mathrm{IAA}(\mathrm{NaI})=\mathrm{DHB}(\mathrm{LiCl})>\mathrm{IAA}(\mathrm{LiCl}) \\
& >\mathrm{HABA}(\mathrm{LiCl})=\mathrm{DHB}(\mathrm{NaI})>\mathrm{DT}(\mathrm{LiCl}) \\
& >\mathrm{HABA}(\mathrm{NaI}) .
\end{aligned}
$$

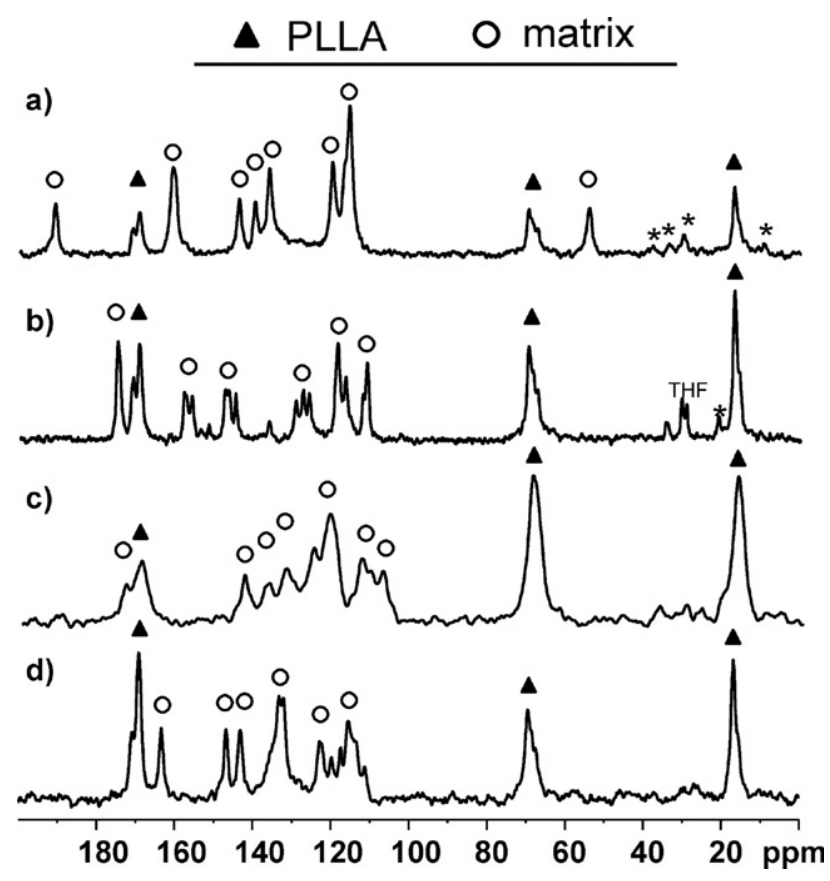

Figure 2. ${ }^{13} \mathrm{C} \mathrm{CP} / \mathrm{MAS}$ spectra of the MALDI mixture in the presence of $\mathrm{NaI}$ (polymer:matrix:NaI 1:10:1) crystallized from THF recorded with $8 \mathrm{kHz}$ spinning rate at room temperature: (a) DT, (b) DHB, (c) IAA, (d) HABA. Circles represent NMR signals of matrix, the filled triangles represent PLLA ( ${ }^{*}$-spinning sidebands).

\section{${ }^{13}$ C NMR Study of PLLA/DPE with MALDI-TOF Matrices}

Detailed analysis of solid-state NMR spectra of crystallized DT, DHB, HABA, and IAA compounds and matrices recrystallized in THF with the addition of $\mathrm{NaI}$ or $\mathrm{LiCl}$ as well as the spectra for PLLA/DPE pure polymer are available as Supplementary Materials, which can be found in the electronic version of this article. We recorded the ${ }^{13} \mathrm{C} \mathrm{CP} / \mathrm{MAS}$ spectra for the four matrices with PLLA/DPE and NaI as the cationization agent (Figure 2) and the appropriate ${ }^{13} \mathrm{CP} / \mathrm{MAS}$ spectra with $\mathrm{LiCl}$ salt (Figure 3).

We analyzed the ${ }^{13} \mathrm{C}$ data to establish the influence of the matrix-salt mixture on the morphology of the polylactide (and vice versa). We evaluated the change of line-shape of the matrix signals and the ratio of components for the carboxyl group which reflects the crystallinity of polymer. Comparison of spectra for PLLA/

\begin{tabular}{|c|c|c|c|c|c|c|c|c|}
\hline \multirow{3}{*}{$\begin{array}{l}\text { Laser power } \\
\text { arbitrary units }\end{array}$} & \multicolumn{8}{|c|}{ Average S/N } \\
\hline & \multicolumn{4}{|c|}{$\mathrm{Nal}$} & \multicolumn{4}{|c|}{$\mathrm{LiCl}$} \\
\hline & DHB & DT & HABA & IAA & DHB & DT & HABA & IAA \\
\hline 1800 & 247 & 444 & 214 & 420 & 379 & 189 & 217 & 285 \\
\hline 1900 & 220 & 438 & 174 & 389 & 392 & 240 & 242 & 259 \\
\hline 2000 & 180 & 442 & 209 & 362 & 352 & 217 & 278 & 254 \\
\hline
\end{tabular}
DPE:DT:salt revealed significant differences in the

Table 1. S/ $\mathrm{N}$ values for PLLA in different matrices in the presence of $\mathrm{NaI}$ and $\mathrm{LiCl}$ cationization agents 


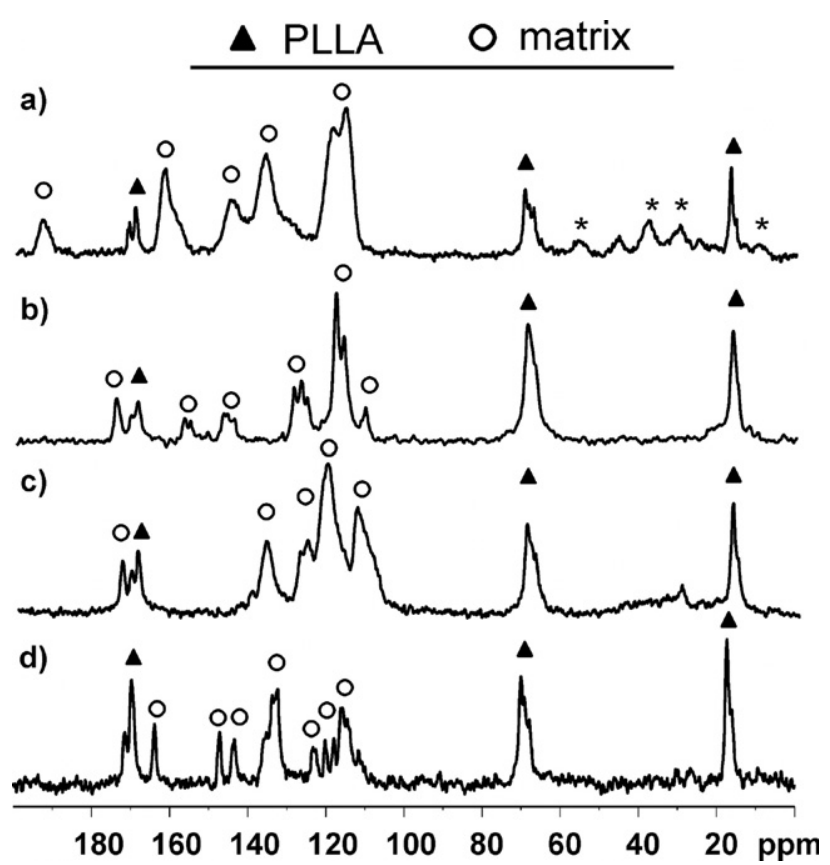

Figure 3. ${ }^{13} \mathrm{C} \mathrm{CP} / \mathrm{MAS}$ spectra of MALDI mixture in presence of $\mathrm{LiCl}$ (polymer:matrix: $\mathrm{LiCl}$ 1:10:1) crystallized from THF recorded with $8 \mathrm{kHz}$ spinning rate at room-temperature: (a) DT, (b) DHB, (c) IAA, (d) HABA. Circles represent NMR signals of matrix, the filled triangles represent PLLA. ( ${ }^{*}$-spinning sidebands).

morphology between samples crystallized with $\mathrm{NaI}$ and $\mathrm{LiCl}$ (Figures 2a and 3a). For the latter case, the resolution was worse and signals were much broader, as is typical for amorphous phases. However, no decisive influence of the matrix and salt on the polymer structure was evident. In both cases, the line-shapes of the carboxyl groups were similar and the proportion of components was roughly the same. Notably, the carboxyl signals were not as well resolved as for the pure polymer (see the Supplementary Materials). Hence, we can only compare the two components corresponding to amorphous and crystalline phases, and in all cases the ratio was 1:2.

The sample prepared with DHB as the matrix and $\mathrm{LiCl}$ as the cationization agent (Figure $3 \mathrm{~b}$ ) showed lower resolution than the sample with $\mathrm{NaI}$ (Figure $2 b$ ), but the effect was not as pronounced as in the case with DT. Based on the data presented in the Supplementary Material, IAA with the polymer formed amorphous phases. Very broad ${ }^{13} \mathrm{C}$ NMR lines were seen for both cationization agents, but resolution of the sample with $\mathrm{LiCl}$ (Figure 3c) was slightly better. In this case, it was possible to deconvolute the carboxyl signals of PLLA. For the sample with NaI (Figure 2c), the carboxyl signals are overlapped and analysis is ambiguous. The spectra for PLLA with HABA with $\mathrm{NaI}$ (Figure 2d) and with $\mathrm{LiCl}$ (Figure $3 \mathrm{~d}$ ) are similar. The ${ }^{13} \mathrm{C}$ signals representing HABA are very similar to those reported elsewhere [16].
In the next step of our project, we were interested in the influence of the matrix on subtle NMR parameters, e.g., ${ }^{13} \mathrm{C}$ chemical shift tensor (CST) elements. For the pure polymer ${ }^{13} \mathrm{C}$ CST data are reported in the Supplementary Materials. Investigations of CST for mixtures of PLLA:matrix:salt were difficult. At low spinning speed, the overlap between different spinning sidebands is multiplied and analysis of the spectrum is ambiguous since the deconvolution procedure was not unequivocal.

The separation of isotropic and anisotropic parts of the spectrum in systems with heavy overlapping is still a challenge for one dimensional (1D) solid-state NMR spectroscopy [16]. Hence, in our project we have employed a two-dimensional approach (2D PASS sequence) [17]. The 2D PASS NMR experiment involves a series of 16 one-dimensional experiments. Each experiment consists of a sequence of five $\pi / 2$ radio frequency pulses with variable delay periods in between them. After CP (cross polarization), the sample is allowed to freely precess during an evolution period synchronized with sample spinning, after which the experimental data are acquired. At the conclusion, data are Fourier transformed in each dimension to generate the twodimensional spectrum.

Figure 4 shows the 2D NMR spectrum of PLLA:DT: $\mathrm{NaI}$, recorded at $278 \mathrm{~K}$ with the spinning rate of 1500 $\mathrm{Hz}$. By proper data shearing (Figure 4b), the spinning sidebands for each carbon were separable, and we were able to calculate the ${ }^{13} \mathrm{C}$ CST parameters. Clearly, the F2

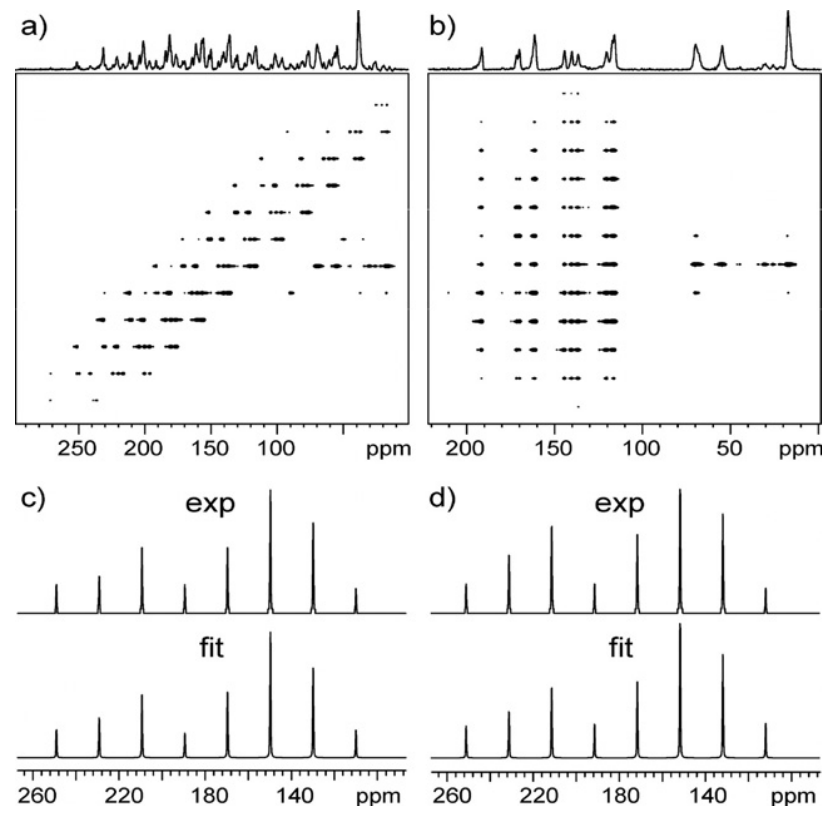

Figure 4. (a) 2D PASS spectrum of PLLA:DT:NaI recorded with spinning rate $1.5 \mathrm{kHz}$, (b) spectrum after data shearing; (c) and (d) show the experimental and the best-fitting simulated 1D CSA sideband pattern for selected carboxyl carbon atoms taken for F1 slices. The spectrum shown (c) represents carboxyl groups at 169 ppm, while the spectrum shown in (d) shows carboxyl groups at $170 \mathrm{ppm}$ 
projection showed only isotropic signals in the spectrum, while F1 represented the chemical shift anisotropy (CSA) of each system. In the present study, we focused attention only on the ${ }^{13} \mathrm{C}$ CST parameters of the carboxyl groups. The appropriate F1 slices and the best fitted simulated spectra are shown in Figure 4c and d. The calculated ${ }^{13} \mathrm{C}$ CST values are collected in the Table in the Supplementary Materials.

For samples prepared for MALDI, measurements of the subtle distinctions between hydrogen bonded domains of polylactide were not seen. This conclusion is based on analyses of ${ }^{13} \mathrm{C} \delta_{\text {ii }}$ parameters available from solid-state NMR measurements and comparison of data for 1 in pure form and embedded in the matrix. It is well known that ${ }^{13} \mathrm{C}$ CST parameters for carboxyl group (in particular ${ }^{13} \mathrm{C} \quad \delta_{22}$ ) are very sensitive to change of strength of hydrogen bonding and analysis of intermolecular interactions [18]. Resemblance of ${ }^{13} \mathrm{C}$ parameters for $\mathbf{1}$ and $\mathbf{1}$ embedded in the MALDI matrix (see Supplementary Materials) justify given supra statement. Analysis of NMR data shown in the Table proved that the matrix and the salt have only slight influence on the molecular structure and the inter-molecular contacts of the polylactide.

\section{Conclusions}

Polylactides are biocompatible and biodegradable polymers with attractive functional properties, for which matrix-assisted laser desorption/ionization-time-offlight mass spectrometry (MALDI-TOF MS) is an important, commonly applied experimental technique used for characterization of molecular mass distribution and degrees of polymerization. However, the quality and reliability of mass spectra strongly depend on experimental conditions and methods of sample preparation. Hence, a full understanding of all the contributions of the experimental variables will allow better control of the analytical processes and is therefore highly desirable.

To the best of our knowledge, we present here the first report showing application of solid-state NMR spectroscopy for the explanation of the nature of polylactide: matrix interactions. Systematic NMR studies of matrices, crystallized under different conditions with addition of two inorganic salts as cationization agents, allowed us to ascertain the changes of the morphology, crystallinity, and amorphicity for compounds, which are commonly used in MALDI-TOF analysis. Advanced analysis of ${ }^{13} \mathrm{C}$ CST NMR parameters for the polymer enabled us to conclude that weak hydrogen bonding is responsible for formation of crystalline domains of polylactide. The ratio of crystalline versus amorphic phases was established employing deconvolution of the carboxylic resonances. Our analysis of actual MALDI-TOF samples by means of NMR spectroscopy permitted us to conclude that under standard crystallization conditions matrices and salts have only slight effects on molecular structure and morphology of the polylactide. The proportions of crystalline and non- crystalline phases were preserved, although subtle distinctions between carboxylic signals in the former phase were observed. Analysis of ${ }^{13} \mathrm{C}$ CST parameters for carboxylic groups of polylactide in the MALDI matrix and their resemblance to parameters established for the pure polymer proved that the polymer chains are in tight contact and this interaction is not disturbed by either the matrix or the salts. Moreover, the crystallinity of the matrix is not a critical step for determining the quality of the MALDITOF analysis, as illustrated by the spectral differences between samples prepared with DT, which is the best matrix for analysis of $\mathbf{1}$, and samples made with IAA, which is slightly worse as a matrix. From the NMR study, clearly DT preserved its crystalline form whilst IAA was very amorphic.

\section{References}

1. Montaudo, G.; Samperi, F.; Montaudo M. MALDI Time-of-Flight Mass Spectrometry of Synthetic Polymers. Prog. Polym. Sci. 2006, 31, 277-357.

2. Mourey, T. H.; Hoteling, A. J.; Balke, S. T.; Owens, K. G. Molar Mass Distributions of Polymers from Size Exclusion Chromatography and Matrix-Assisted Laser Desorption/Ionization Time-of-Flight Mass Spectrometry: Methods for Comparison. J. Appl. Polym. Sci. 2005, 97, 627-639.

3. (a) Nielen, M. W. F. MALDI Time-of-Flight Mass Spectrometry of Synthetic Polymers. Mass Spectrom. Rev. 1999, 18, 309-344. (b) Hanton, S. Mass Spectrometry of Polymers and Polymer Surfaces. Chem. Rev. 2001, 101, 527-569. (c) Macha, S.; Limbach, P. Matrix-Assisted Laser Desorption/Ionization (MALDI) Mass Spectrometry of Polymers. Curr. Opin. Solid State M 2002, 6, 213-220.

4. Hoteling, A. J.; Mourey, T. H.; Owens, K. G. Importance of Solubility in the Sample Preparation of Poly(Ethylene Terephthalate) for MALDITOF-MS. Anal. Chem. 2005, 77, 750-756.

5. Hoteling, A. J.; Erb, W. J.; Tyson, R. J.; Owens, K. G. Exploring the Importance of the Relative Solubility of Matrix and Analyte in MALDI Sample Preparation Using HPLC. Anal. Chem. 2004, 76, 5157-5164.

6. Hoteling, A. J.; Owens, K. G. Improved PSD and CID on a MALDITOF-MS. I. Am. Soc. Mass Spectrom. 2004, 15, 523-535.

7. Creaser, C. S.; Reynolds, J. C.; Hoteling, A. J.; Nichols, W. F.; Owens, K. G. Atmospheric Pressure Matrix-Assisted Laser Desorption/ Ionization Ion Trap Mass Spectrometry of Synthetic Polymers: A Comparison with Vacuum Matrix-Assisted Laser Desorption/Ionization Timeof-Flight Mass Spectrometry. Eur. J. Mass Spectrom. 2003, 9, 33-44.

8. Hoteling, A. J.; Kawaoka, K.; Goodberlet, M. C.; Yu, W. M.; Owens K. G. Optimization of Matrix-Assisted Laser Desorption/Ionization Time-ofFlight Collision-Induced Dissociation Using Poly(Ethylene Glycol). Rapid Commun. Mass Spectrom. 2003, 17, 1671-1676.

9. Meier, M.; Schubert U. Evaluation of a new Multilayer Spotting Technique for MALDI-TOF-MS of Synthetic polymers. Rapid Commun. Mass Spectrom. 2003, 17, 713-716.

10. Hoteling, A. J.; Nichols, W. F.; Giesen, D. J.; Lenhard, J. R.; Knochenmuss R. Electron Transfer Reactions in Laser Desorption/Ionization and Matrix-Assisted Laser Desorption/Ionization: Factors Influencing Matrix and Analyte Ion Intensities. Eur. J. Mass Spectrom. 2006, 12, 345-358.

11. Biela T.; Duda A.; Pasch H.; Rode K. Star-Shaped Poly(l-Lactide)s with Variable Numbers of Hydroxyl Groups at Polyester Arms Chain-Ends and Directly Attached to the Star-Shaped Core-Controlled Synthesis and Characterization J. Polym. Sci. Pol. Chem. Part A 2005, 43, 6116-6133.

12. Morcombe, C. R.; Zilm, K. W. Chemical Shift Referencing in MAS Solid State NMR. J. Magn. Reson. 2003, 162, 479-486.

13. Metz, G.; Wu, X.; Smith, S. O. Ramped-Amplitude Cross Polarization in Magic Angle Spinning NMR. J. Magn. Reson. 1994, 110, 219-227.

14. Bennett, A. W.; Rienstra, C. M.; Auger, M.; Lakshmi, K. V.; Griffin, R. G. Heteronuclear Decoupling in Rotating Solids. J. Chem. Phys. 1995, 103, 6951-6958.

15. Win-NMR, Bruker-Franzen Analytik GmbH, Version 6.0, Bremen, Germany, 1993.

16. (a) Hu, J. Z.; Wang, W.; Liu, F.; Solum, M. S.; Alderman, D. W.; Pugmire, R. J. Magic Angle Turning Experiments for Measuring Chemical-ShiftTensor Principal Values in Powdered Solids. J. Magn. Reson. 1995, 113, 210-222. (b) Alderman, D. W.; McGeorge, G.; Hu, J. Z.; Pugmire, R. J.; Grant D. M. A Sensitive, High Resolution Magic Angle Turning Experiment for Measuring Chemical Shift Tensor Principal Values. Mol. Phys. 1998, 95, 1113-1126. (c) Frydman, L.; Chingas, G. C.; Lee, Y. K.; Grandinetti, P. J.; Eastman, M. A.; Barral, G. A.; Pines, A. Correlation of Isotropic and Anisotropic Chemical Shifts in Solids by Two-Dimensional 
Variable Angle Spinning NMR. Israel J. Chem. 1992, 32, 161-164. (d) Kolbert, A. C.; Griffin, R. G. Two-Dimensional Resolution of Isotropic and Anisotropic Chemical Shifts in Magic Angle Spinning NMR. Chem. Phys. Lett. 1990, 166, 87-91.

17. (a) Antzutkin, O. N. Sideband Manipulation in Magic Angle Spinning Nuclear Magnetic Resonance. Prog. Nucl. Magn. Reson. Sp. 1999, 35,
203-266. (b) Antzutkin, O. N.; Shekar, S. C.; Levitt, H. M. Two-dimensional sideband separation in magic angle spinning NMR. J. Magn. Reson. 1995, 115, 7-19.

18. Potrzebowski, M. J. What High-Resolution Solid-State NMR Spectroscopy Can Offer to Organic Chemists. Eur. J. Org. Chem. 2003, 8, 1367-1376. 